VoL. 55 (1997) [497-501]

\title{
A CHARACTERISATION OF WEAK COMPACTNESS IN BANACH SPACES
}

\author{
WARREN B. MOORS
}

In this short note we give a new characterisation of weak compactness.

The primary purpose of this paper is to provide a proof of the 'Note added in proof' in [3]. In doing so, we shall also derive a new characterisation of weak compactness in Banach spaces.

For a Banach space $X$ with closed unit ball $B(X)$, we shall denote by ext $B\left(X^{*}\right)$, the set of all extreme points of the dual ball $B\left(X^{*}\right)$ and we shall denote by $\sigma_{e}$, the weak topology on $X$ generated by $\operatorname{ext} B\left(X^{*}\right)$.

Observe that the $\sigma_{e}$-topology on $X$ is Hausdorff and that the closed unit ball $B(X)$ is also closed in the $\sigma_{e}$-topology. Both of these observations follow from the fact that for each $x \in X$, there exists an element $e \in \operatorname{ext} B\left(X^{*}\right)$ such that $e(x)=\|x\|$. In the case when $X \equiv C(K)-(K$ compact and Hausdorff and $B(X)$ is the supremum norm ball), the $\sigma_{e}$-topology is the pointwise topology on $C(K)$. In the case of a Banach space $X$, whose dual ball $B\left(X^{*}\right)$ is rotund, the $\sigma_{e}$-topology is the weak topology on $X$.

TheOREM 0.1. (Rainwater's theorem, [5]) Let $X$ be a Banach space and let $\left\{x_{n}: n \in N\right\}$ be a bounded sequence in $X$. Then $\left\{x_{n}: n \in N\right\}$ converges weakly to $x$ if and only if $\left\{x_{n}: n \in N\right\}$ converges to $x$ in the $\sigma_{e}$-topology.

Corollary 0.1. A bounded sequence in a Banach space $X$ is weakly Cauchy if and only if it is $\sigma_{e}$-Cauchy.

Proof: It need only be observed that a sequence $\left\{x_{n}: n \in N\right\}$ is weakly Cauchy if and only if for each pair $\left\{k_{n}: n \in N\right\}$ and $\left\{j_{n}: n \in N\right\}$ of increasing sequences of natural numbers, the sequence $\left\{x_{k_{n}}-x_{j_{n}}: n \in N\right\}$ converges weakly to 0 .

ThEOREM 0.2. Let $F$ be an infinite bounded subset of a Banach space $X$. Then there exists a countably infinite subset $E$ of $F$ such that $\overline{c o}^{\sigma_{e}} E=\overline{c o} E$. In particular,

Received 9th July, 1996.

Research supported by a New Zealand Science and Technology Post-Doctoral Fellowship.

Copyright Clearance Centre, Inc. Serial-fee code: 0004-9729/97 \$A2.00+0.00. 
every bounded sequence $\left\{x_{n}: n \in N\right\}$ in $X$ possesses a subsequence $\left\{x_{n_{k}}: k \in N\right\}$ such that $\overline{c o}^{\sigma_{e}}\left\{x_{n_{k}}: k \in N\right\} \subseteq \overline{c o}\left\{x_{n}: n \in N\right\}$.

Proof: Suppose that this is not the case. Then for each countably infinite subset $E$ of $F$ there exists an element $z \in \overline{c o}^{\sigma_{e}} E \backslash \overline{c o} E$. Using this, let us show that for any infinite sequence $\left\{x_{n}: n \in N\right\}$ in $F$, there exists an element

$$
z \in\left(\bigcap_{n=1}^{\infty} \overline{c o}^{\sigma}{ }^{\alpha}\left\{x_{k}: k \geqslant n\right\}\right) \backslash \overline{c o}\left\{x_{n}: n \in N\right\}
$$

We begin by observing that since $B(X)$ is $\sigma_{e}$-closed and convex, we have that $\operatorname{diam} F=$ diam $\overline{c o}^{\sigma_{e}} F$. So, in particular, we have that $\overline{c o}^{\sigma_{e}} F$ is also bounded. Let $\left\{x_{n}: n \in N\right\}$ be an infinite sequence in $F$. By our assumption, the Bishop-Phelps theorem and a separation argument, there exists $f \in X^{*}$ and $z \in \overline{c o}^{\sigma_{e}}\left\{x_{n}: n \in N\right\}$ such that

$$
f(z)=\max f\left(\overline{c o}^{\sigma_{e}}\left\{x_{n}: n \in N\right\}\right)>\sup f\left(\overline{c o}\left\{x_{n}: n \in N\right\}\right) .
$$

We claim that

$$
z \in \bigcap_{n=1}^{\infty} \overline{c o}^{\sigma_{e}}\left\{x_{k}: k \geqslant n\right\} .
$$

To see this, we note that for each $n \in N$,

$$
\overline{c o}^{\sigma_{e}}\left\{x_{k}: k \in N\right\}=\operatorname{co}\left(\overline{c o}^{\sigma_{e}}\left\{x_{k}: k>n\right\} \cup \operatorname{co}\left\{x_{1}, x_{2}, \ldots, x_{n}\right\}\right) .
$$

Therefore, for each $n \in N$, there exists $\lambda_{n} \in[0,1], z_{n} \in \overline{c o}^{\sigma_{e}}\left\{x_{k}: k>n\right\}$ and $y_{n} \in \operatorname{co}\left\{x_{1}, x_{2},,, x_{n}\right\}$ such that $z=\lambda_{n} z_{n}+\left(1-\lambda_{n}\right) y_{n}$. It now follows that for each $n \in N, \lambda_{n}=1$ and $z=z_{n} \in \overline{c o}^{\sigma_{e}}\left\{x_{k}: k>n\right\}$ and so

$$
z \in\left(\bigcap_{n=1}^{\infty} \overline{c o}^{\sigma_{e}}\left\{x_{k}: k \geqslant n\right\}\right) \backslash \overline{c o}\left\{x_{n}: n \in N\right\} .
$$

Let $\left\{x_{n}: n \in N\right\}$ be an arbitrary sequence of distinct elements of $F$. We shall show that $\left\{x_{n}: n \in N\right\}$ contains a weakly Cauchy subsequence. Let $Y \equiv \overline{s p}\left\{x_{n}\right.$ : $n \in N\} \subseteq X$ and denote by $E_{Y} \equiv \operatorname{ext} B\left(Y^{*}\right)$. Since $\left(B\left(Y^{*}\right)\right.$, weak $)$ is separable and metrisable so is $\left(E_{Y}\right.$, weak $\left.{ }^{*}\right)$. Let $\left\{e_{Y}^{n}: n \in N\right\}$ be a dense subset of $E_{Y}$. Since for each $m \in N$, the sequence $\left\{e_{Y}^{m}\left(x_{n}\right): n \in N\right\}$ is bounded, we may apply a Cantor diagonalisation argument to extract a subsequence $\left\{x_{n_{k}}: k \in N\right\}$ of $\left\{x_{n}: n \in N\right\}$ such that

$$
\lim _{k \rightarrow \infty} e_{Y}^{m}\left(x_{n_{k}}\right) \text { exists for each } m \in N \text {. }
$$

We claim that the sequence $\left\{x_{n_{k}}: k \in N\right\}$ is weakly Cauchy. To see this, we consider the following. For each $k \in N$, define $\widehat{x}_{n_{k}}: E_{Y} \rightarrow R$ by $\widehat{x}_{n_{k}}\left(e_{Y}\right) \equiv e_{Y}\left(x_{n_{k}}\right)$. 
Clearly, each $\widehat{x}_{n_{k}}$ is continuous on $\left(E_{Y}\right.$, weak $\left.{ }^{*}\right)$. We shall show that the sequence $\left\{\widehat{x}_{n_{k}}: k \in N\right\}$ is pointwise convergent to some continuous function $\widehat{z}_{0}$ on $\left(E_{Y}\right.$, weak $\left.{ }^{*}\right)$; and then apply Rainwater's theorem. Now, for each $x \in \overline{c o}^{\sigma_{e}}\left\{x_{n}: n \in N\right\}$ we may define a continuous 'lift' $\widehat{x}$ of $x$ onto $E_{Y}$ in the following manner. Define $\widehat{x}: E_{Y} \rightarrow R$, by $\widehat{x}\left(e_{Y}\right) \equiv e(x)$ where $e$ is any member of $B\left(X^{*}\right)$ such that $\left.e\right|_{Y} \equiv e_{Y}$. Our first task is to show that $\widehat{x}$ is well-defined. For each $e_{Y} \in E_{Y}$, let $H B\left(e_{Y}\right) \equiv\left\{e \in B\left(X^{*}\right):\left.e\right|_{Y}=e_{Y}\right\}$. It is not too hard to show that the set-valued mapping $e_{Y} \rightarrow H B\left(e_{Y}\right)$ is a weak ${ }^{*}$ cusco on $E_{Y}$, that is, for each $e_{Y} \in E_{Y}, H B\left(e_{Y}\right)$ is non-empty, weak* compact and convex and $e_{Y} \rightarrow H B\left(e_{Y}\right)$ is weak ${ }^{*}$ upper semi-continuous. Furthermore, it is not too hard to see that each $H B\left(e_{Y}\right)$ is an extremal subset of $B\left(X^{*}\right)$ and so ext $H B\left(e_{Y}\right) \subseteq \operatorname{ext} B\left(X^{*}\right)$. Let us also note that by the Krein-Milman theorem, $H B\left(e_{Y}\right)={\overline{c \sigma^{*}}}^{\omega^{*}}$ ext $H B\left(e_{Y}\right)$, for each $e_{Y} \in E_{Y}$. So to show that $\widehat{x}$ is well-defined it suffices to show that if $e_{1}$ and $e_{2} \in \operatorname{ext} H B\left(e_{Y}\right)$ then $e_{1}(x)=e_{2}(x)$ or, equivalently, show that $x \in \operatorname{ker}\left(e_{1}-e_{2}\right)$. However, this is obvious since $\left\{x_{n}: n \in N\right\} \subseteq \operatorname{ker}\left(e_{1}-e_{2}\right)$ and $\operatorname{ker}\left(e_{1}-e_{2}\right)$ is $\sigma_{e^{-}}$ closed and convex. The fact that $\widehat{x}$ is continuous on $\left(E_{Y}\right.$, weak $\left.{ }^{*}\right)$ follows directly from the fact that $e_{Y} \rightarrow H B\left(e_{Y}\right)$ is a weak ${ }^{*}$ cusco on $E_{Y}$. Choose

$$
z_{0} \in\left(\bigcap_{m=1}^{\infty} \overline{c o}^{\sigma_{e}}\left\{x_{n_{k}}: k \geqslant m\right\}\right) \backslash \overline{c o}\left\{x_{n_{k}}: k \in N\right\}
$$

We shall show that $\left\{\widehat{x}_{n_{k}}: k \in N\right\}$ converges pointwise to $\widehat{z}_{0}$ on $E_{Y}$. Let us first observe that for each $e_{Y} \in E_{Y}$

$$
\widehat{z}_{0}\left(e_{Y}\right) \in \bigcap_{m=1}^{\infty} \overline{c o}\left\{\widehat{x}_{n_{k}}\left(e_{Y}\right): k \geqslant m\right\} .
$$

Hence, for each $m \in N$,

$$
\widehat{z}_{0}\left(e_{Y}^{m}\right)=\lim _{k \rightarrow \infty} \widehat{x}_{n_{k}}\left(e_{Y}^{m}\right) .
$$

Next, suppose that for some $e_{Y}^{\prime} \in E_{Y}$,

$$
\lim _{k \rightarrow \infty} \widehat{x}_{n_{k}}\left(e_{Y}^{\prime}\right) \neq \widehat{z}_{0}\left(e_{Y}^{\prime}\right)
$$

Then there exists an $\varepsilon>0$ and a subsequence $\left\{x_{n_{k_{l}}}: l \in N\right\}$ of $\left\{x_{n_{k}}: k \in N\right\}$ such that $\left|\widehat{z}_{0}\left(e_{Y}^{\prime}\right)-\widehat{x}_{n_{k_{l}}}\left(e_{Y}^{\prime}\right)\right|>\varepsilon$ for all $l \in N$. Moreover, by possibly replacing $e_{Y}^{\prime}$ by $-e_{Y}^{\prime}$ and passing to a subsequence, we may assume that $\widehat{z}_{0}\left(e_{Y}^{\prime}\right)<\widehat{x}_{n_{k_{l}}}\left(e_{Y}^{\prime}\right)-\varepsilon$ for each $l \in N$. Now choose

$$
x_{0} \in\left(\bigcap_{m=1}^{\infty} \overline{\operatorname{co}}^{\sigma_{e}}\left\{x_{n_{k_{l}}}: l \geqslant m\right\}\right) \backslash \overline{c o}\left\{x_{n_{k_{l}}}: l \in N\right\} .
$$


As before, we have that

$$
\widehat{x}_{0}\left(e_{Y}\right) \in \bigcap_{m=1}^{\infty} \overline{c o}\left\{\widehat{x}_{n_{k_{l}}}\left(e_{Y}\right): l \geqslant m\right\} .
$$

Therefore $\widehat{x}_{0}\left(e_{Y}^{\prime}\right) \geqslant \widehat{z}_{0}\left(e_{Y}^{\prime}\right)+\varepsilon$ while $\widehat{x}_{0}\left(e_{Y}^{m}\right)=\widehat{z}_{0}\left(e_{Y}^{m}\right)$ for each $m \in N$. But this is not possible, since both $\widehat{x}_{0}$ and $\widehat{z}_{0}$ are continuous on $\left(E_{Y}\right.$, weak $\left.{ }^{*}\right)$. Hence we must have that $\widehat{z}_{0}$ is the pointwise limit of the sequence $\left\{\widehat{x}_{n_{k}}: k \in N\right\}$. Therefore, by Corollary $0.1,\left\{x_{n_{k}}: k \in N\right\}$ is weakly Cauchy in $Y$ and so weakly Cauchy in $X$. The next and penultimate step is to show that the sequence $\left\{x_{n_{k}}: k \in N\right\}$ converges weakly to $z_{0}$. We know from Rainwater's theorem that to do this we need only show that

$$
\lim _{k \rightarrow \infty} e\left(x_{n_{k}}\right)=e\left(z_{0}\right) \quad \text { for each } e \in \operatorname{ext} B\left(X^{*}\right)
$$

To this end, let $e \in \operatorname{ext} B\left(X^{*}\right)$. Now, $\left\{e\left(x_{n_{k}}\right): k \in N\right\}$ is a Cauchy sequence in $\mathrm{R}$ and

$$
e\left(z_{0}\right) \in \bigcap_{m=1}^{\infty} \overline{c o}\left\{e\left(x_{n_{k}}\right): k \geqslant m\right\} \text {. }
$$

Therefore,

$$
e\left(z_{0}\right)=\lim _{k \rightarrow \infty} e\left(x_{n_{k}}\right) .
$$

This shows that $z_{0}$ is the weak limit of $\left\{x_{n_{k}}: k \in N\right\}$. The final step is to observe that this gives rise to our desired contradiction, since we chose $z_{0} \notin \overline{c o}\left\{x_{n_{k}}: k \in N\right\}$.

COROLlary 0.2 . Every bounded sequence $\left\{x_{n}: n \in N\right\}$ in a Banach space $X$ contains a subsequence $\left\{x_{n_{k}}: k \in N\right\}$ such that

$$
\bigcap_{m=1}^{\infty} \overline{c o}^{\sigma_{e}}\left\{x_{n_{k}}: k \geqslant m\right\} \subseteq \bigcap_{m=1}^{\infty} \overline{c o}\left\{x_{n}: n \geqslant m\right\} .
$$

Proof: This follows from the previous theorem and a diagonalisation argument. $]$

The next corollary improves the main result in [1].

COROLlary 0.3. A bounded subset $F$ of a Banach space $X$ is relatively weakly compact if and only if for each sequence $\left\{x_{n}: n \in N\right\}$ in $F$

$$
\bigcap_{m=1}^{\infty} \overline{c o}^{\sigma_{e}}\left\{x_{n}: n \geqslant m\right\} \neq \emptyset .
$$

In particular, $F$ is relatively weakly compact if and only if $F$ is relatively $\sigma_{e}$-countably compact.

Proof: The proof of this follows from Corollary 0.2 and the fact that $F$ is relatively weakly compact if and only if for each sequence $\left\{x_{n}: n \in N\right\}$ in $F$

$$
\bigcap_{m=1}^{\infty} \bar{c}\left\{x_{n}: n \geqslant m\right\} \neq \emptyset \text {. }
$$


REMARK. The author benefitted greatly by having an acquaintance with the manuscript [4], which contains a simplification of the main result in [2].

\section{REFERENCES}

[1] J. Bourgain and M. Talagrand, 'Compacité Extrêmale', Proc. Amer. Math. Soc. 80 (1980), 68-70.

[2] S.S. Khurana, 'Pointwise compactness on extreme points', Proc. Amer. Math. Soc. 83 (1981), 347-348.

[3] W.B. Moors, 'A selection theorem for weak upper semi-continuous set-valued mappings', Bull. Austral. Math. Soc. 53 (1996), 213-227.

[4] I. Namioka, 'A simplification of Khurana's proof', (unpublished manuscript, 1981).

[5] J. Rainwater, 'Weak convergence of bounded sequences', Proc. Amer. Math. Soc. 14 (1963), p. 999.

Department of Mathematics

The University of Auckland

Private Bag 92019

Auckland

New Zealand 